\title{
Universiteit
}

Leiden

The Netherlands

\section{Effect of spatial filtering on the Schmidt decomposition of entangled} photons

Exter, M.P. van; Aiello, A.; Oemrawsingh, S.S.R.; Nienhuis, G.; Woerdman, J.P.

\section{Citation}

Exter, M. P. van, Aiello, A., Oemrawsingh, S. S. R., Nienhuis, G., \& Woerdman, J. P. (2006).

Effect of spatial filtering on the Schmidt decomposition of entangled photons. Physical Review A, 74, 012309. doi:10.1103/PhysRevA.74.012309

Version: $\quad$ Not Applicable (or Unknown)

License: $\quad$ Leiden University Non-exclusive license

Downloaded from: https://hdl.handle.net/1887/61324

Note: To cite this publication please use the final published version (if applicable). 


\title{
Effect of spatial filtering on the Schmidt decomposition of entangled photons
}

\author{
M. P. van Exter, A. Aiello, S. S. R. Oemrawsingh, G. Nienhuis, and J. P. Woerdman \\ Huygens Laboratory, Leiden University, P. O. Box 9504, 2300 RA Leiden, The Netherlands
}

(Received 22 December 2005; published 13 July 2006)

\begin{abstract}
A simple analytic decomposition of the spatially entangled two-photon field allows us to generalize the earlier results of Law and Eberly [Phys. Rev. Lett. 92, 127903 (2004)] to more realistic experimental geometries of spontaneous parametric down-conversion. We quantify analytically how spatial filtering reduces the Schmidt number or dimensionality of the two-photon entanglement from a "generated" value to a generally much lower "usable or detected" value, for both collinear and noncollinear phase matching. We also discuss the intimate relation between the (two-photon) Schmidt number and the classical (one-photon) concept of etendue or geometric extent.
\end{abstract}

DOI: 10.1103/PhysRevA.74.012309

\section{INTRODUCTION}

Quantum entanglement of two inseparable quantum systems comes in many flavors. The simplest form of entanglement occurs for two-level systems, where the entanglement of the corresponding qubits is well studied and well understood, in particular via its nonclassical correlations known as the Bell inequalities [1]. Quantum systems with more levels, characterized by qudits or quNits, can exhibit more complicated and conceptually more interesting high-dimensional forms of entanglement. High-dimensional entanglement can be applied, among other ways, in quantum communication protocols that are more robust against decoherence and eavesdropping attacks [2]. A powerful tool to characterize any form of entanglement is the so-called Schmidt decomposition [3], in which the quantum state of the combined system is written as a sum over biorthogonal product states of the two subsystems. Unfortunately, this decomposition is often difficult to find, especially in systems with continuous variables and infinitely many levels.

Quantum-entangled photon pairs can be easily generated with the nonlinear optical process of spontaneous parametric down-conversion (SPDC) [4], in which a pump photon splits into two photons of lower energy. Depending on the specific geometry the two generated photons are simultaneously entangled in (i) polarization, (ii) time or frequency, and (iii) transverse position or momentum. Just as the generated polarization entanglement has served as a prototype for the two-dimensional entanglement of (polarization) qubits, the generated spatial entanglement can serve as a prototype for the high-dimensional entanglement of (spatial) qudits. This form of entanglement is rapidly attracting more interest, with experimental demonstrations of, among other things, entanglement of photon angular momentum [5-10], very small values of the product of the position and momentum uncertainties, $\Delta x_{-} \Delta k_{+} \ll 1[11]$, and "pixel entanglement" based on multiple detectors [12] or segmented screens [13].

An exact treatment of the spatial entanglement generated with SPDC is extremely difficult, in particular due to the role of phase matching. Drastic approximations are needed to simplify the problem. Several groups have chosen to concentrate on the angular momentum of the photon and single out the fundamental $p=0$ radial modes $[5,6]$. A Schmidt decomposition in this limited basis demonstrates the entanglement of the photon angular momenta. A more complete decompo-
PACS number(s): 03.67.Mn, 42.50.Dv, 42.65.Lm

sition (for collinear phase matching) provides for additional parity relations [7].

Recently, Law and Eberly [14] gave an elegant analytic description of the complete modal decomposition of a spatially entangled two-photon field for the case of collinear phase matching. After briefly summarizing their result, we will extend this description beyond the case of collinear phase matching, since this is rarely used in experiments, and derive expressions that are more realistic and thus more useful for experimentalists. This allows us to show that the number of entangled spatial modes that is detected and usable in a practical setup is usually much smaller than the generated number of modes that was calculated in Ref. [14]. If the collection angle of the detection system is too small to image the full generated space angle, as is often the case in experiments, the size of the entangled phase space can easily be reduced by several orders of magnitude. After extending the description to the case of noncollinear phase matching, we make a link between the two-photon Schmidt number [3] and the classical (one-photon) concept of etendue $[15,16]$.

\section{SCHMIDT DECOMPOSITION OF THE TWO-PHOTON STATE}

\section{A. Generated Schmidt number}

The analytic description of spatial entanglement is relatively simple if one considers only the spatial degrees of freedom, by working in the monochromatic limit, and uses a Gaussian approximation for the phase-matching condition. Following the notation of Law and Eberly [14], we write the two-photon probability amplitude $C_{g}(\mathbf{k}, \mathbf{q})$ for generating signal and idler photons with transverse momenta $\mathbf{k}$ and $\mathbf{q}$ as

$$
C_{g}(\mathbf{k}, \mathbf{q}) \equiv\langle\mathbf{k}, \mathbf{q} \mid \Psi\rangle \propto e^{-|\mathbf{k}+\mathbf{q}|^{2} / \sigma^{2}} e^{-b^{2}|\mathbf{k}-\mathbf{q}|^{2}} .
$$

The first factor in this expression originates from the transverse momentum profile $\mathcal{E}_{p}\left(\mathbf{q}_{p}\right)=\exp \left(-\left|\mathbf{q}_{p}\right|^{2} / \sigma^{2}\right)$ of a Gaussian pump beam with a real-space beam width $w_{0}=2 / \sigma$. The second factor originates from a (rough) Gaussian expansion of the phase-matching condition, which for the collinear type-I geometry reads $[14,17] \quad C_{P M}(\mathbf{k}, \mathbf{q})=\operatorname{sinc}\left(\frac{1}{2} \Delta k_{z} L\right)$ $\approx \operatorname{sinc}\left(b^{2}|\mathbf{k}-\mathbf{q}|^{2}\right) \approx \exp \left(-b^{2}|\mathbf{k}-\mathbf{q}|^{2}\right)$, where $b^{2}=L /\left(4 k_{p}\right)$ and $k_{p}=n_{p} \omega_{p} / c$ is the momentum of the pump photons inside the crystal of length $L$. As a side remark we note that the usual 
anticorrelation of the transverse momenta $\mathbf{k} \approx-\mathbf{q}$ applies only to the weak-focusing limit $(b \sigma \ll 1)$; strong focusing $(b \sigma \gg 1)$ yields a correlation instead, with $\mathbf{k} \approx \mathbf{q}$.

For a Schmidt decomposition of the Gaussian two-photon amplitude function $C(\mathbf{k}, \mathbf{q})$, one needs to find the two orthogonal sets of signal and idler functions for which the continuous expression (1) turns into a discrete sum over simple product functions. Our Schmidt decomposition is based upon a mathematical relation that, quite surprisingly, also plays an essential role in the description of the effect of velocitychanging collisions in gas-phase laser spectroscopy and in classical transport theory [18-20]. We hereby refer in particular to the observation of Snider [18] that the HermiteGaussian modes are eigenmodes of the Keilson-Storer collision kernel $W\left(\mathbf{v}^{\prime} \rightarrow \mathbf{v}\right) \propto \exp \left(\left|\mathbf{v}-\alpha \mathbf{v}^{\prime}\right|^{2} / \delta^{2}\right)$, which quantifies the probability rate of velocity changes from $\mathbf{v}^{\prime}$ to $\mathbf{v}, \delta$ being proportional to the mean velocity $[19,20]$.

Inspired by Snider's formulation, which is essentially based on the Gaussian generating function of the Hermite polynomials, we found a more symmetric formulation that is ideally suited for the required Schmidt decomposition, but might also be applicable elsewhere. In its simplest onedimensional form this relation is

$$
C(k, q) \propto \exp \left[-\gamma_{0}^{2}\left(k^{2}+q^{2}-2 \beta k q\right)\right] \propto \sum_{n=0}^{\infty} \alpha^{n} u_{n}(\gamma k) u_{n}(\gamma q),
$$

where $u_{n}(\gamma k) \propto H_{n}(\gamma k) \exp \left(-\frac{1}{2} \gamma^{2} k^{2}\right)$ are the normalized Hermite-Gaussian functions. The parameter $-1<\beta<1$ quantifies the "separability" of the composite function $C(k, q)$. The (exponential) distribution over the modal amplitudes $\sqrt{\lambda_{n}}=\alpha^{n}$, with $\alpha=1 / \beta-\sqrt{1 / \beta^{2}-1}$, yields the one-dimensional Schmidt number $K_{1 D}=\left(\Sigma \lambda_{n}\right)^{2} / \Sigma \lambda_{n}^{2}$ $=\left(1+\alpha^{2}\right) /\left(1-\alpha^{2}\right)=1 / \sqrt{1-\beta^{2}}$. The characteristic waist of the separated modes is $\gamma^{2}=2 \gamma_{0}^{2} \sqrt{1-\beta^{2}}$.

In comparing Eqs. (1) and (2) we note that the $x$ and $y$ dependences in Eq. (1) factorize via $C(\mathbf{k}, \mathbf{q})$ $=C\left(k_{x}, q_{x}\right) C\left(k_{y}, q_{y}\right)$, where each factor has the same functional form as Eq. (2); the effective number of modes or the two-dimensional Schmidt number of our biphoton field is thus $K=K_{1 D}^{2}$. A quantitative comparison yields $K_{1 D}$ $=\frac{1}{2}\left(\frac{1}{b \sigma}+b \sigma\right)$ and $\gamma^{2}=4 b / \sigma$, as in Ref. [14].

\section{B. Usable or detected Schmidt number}

The key development is now that either the finite size of the detectors or the practically inevitable apertures generally limits the detection system, so that the usable number of entangled modes can be much lower than the generated number found above. This effect of spatial filtering can be easily calculated if the apertures are positioned in the far field and if their amplitude transmission functions are assumed to have identical Gaussian forms. This gives the apertured biphoton amplitude

$$
C_{a p}(\mathbf{k}, \mathbf{q})=C_{g}(\mathbf{k}, \mathbf{q}) e^{-a^{2}\left(|\mathbf{k}|^{2}+|\mathbf{q}|^{2}\right)},
$$

the same functional form as before. By comparing this expression with the generic form of Eq. (2), we easily find the



FIG. 1. Far-field emission pattern of the four fundamental SPDC geometries, being (type I or type II) and (collinear or noncollinear). In the type-I noncollinear geometry of (c), the three arrows indicate the ring radius $q_{0}$ and the extra horizontal momenta $\delta q_{x}$ and $\delta k_{x}$ of the signal and idler photons, respectively. Two Gaussian apertures, depicted as circles, are located around the far-field points $\left( \pm q_{0}, 0\right)$.

associated $\beta$ parameter and from that the Schmidt number of the filtered spatial entanglement as

$$
K_{2 D}=\frac{\left[(1 / \sigma)^{2}+b^{2}+a^{2}\right]^{2}}{\left[(1 / \sigma)^{2}+b^{2}+a^{2}\right]^{2}-\left[(1 / \sigma)^{2}-b^{2}\right]^{2}} .
$$

We recall that the three parameters $\sigma, 1 / b$, and $1 / a$ quantify the spread in transverse momenta of the pump beam, of the collinear SPDC, and of the aperture selection, respectively. In the absence of apertures, i.e., for $a=0$, Eq. (4) reduces to the previous result [14]. Upon decreasing the aperture size, i.e., for increasing $a$, the detected Schmidt number $K_{2 D}$ will gradually decrease to its asymptotic value $K_{2 D}=1$ for $[a \gg b,(1 / \sigma)]$.

\section{Four different geometries}

Next, we will extend the discussion by further analyzing the geometrical consequences of phase matching. First of all, we note that the earlier expression $\Delta k_{z}=\left(2 b^{2} / L\right)|\mathbf{k}-\mathbf{q}|^{2}$ was only an approximation that basically ignored the birefringence in the generating crystal. If birefringence is included, one should distinguish between the four different geometries that are used in SPDC, being (type-I or type-II) and (collinear or noncollinear) (see Fig. 1). In type-I SPDC, where the signal and idler have the same polarization, the (longitudinal) momentum mismatch is actually [21]

$$
\Delta k_{z}=\left(2 b^{2} / L\right)|\mathbf{k}-\mathbf{q}|^{2}+c\left(k_{y}+q_{y}\right)+d,
$$

where $c$ is the internal walk-off angle of the extraordinary wave, $d$ is a constant that depends on the crystal's cut angle and orientation, and the principal axis of the uniaxial crystal was taken in the $y z$ plane. The extra walk-off terms 
$c\left(k_{y}+q_{y}\right)$ are hardly noticeable in the weak-focusing limit $(b \sigma \ll 1)$, which places a strong restriction on the sum $\mathbf{k}+\mathbf{q}$. In the case of stronger focusing, however, we do expect these terms to play a role [22].

For type-II SPDC, where the signal and idler have orthogonal polarizations, the expression for the momentum mismatch is approximately the same, but lacks the term $c q_{y}$, q being the transverse momentum of the extraordinary light, assuming identical walk-off angles at $\omega_{p}$ and $\omega_{p} / 2$. For type-II SPDC the remaining walk-off term $c k_{y}$ is always important, as it shifts the centers of the ordinary and extraordinary SPDC rings in opposite $(y)$ directions with respect to the pump [see Figs. 1(b) and 1(d)].

Our previous results for the generated and detected Schmidt number applies only to the type-I collinear geometry [Fig. 1(a)]. For the other three geometries the Schmidt decomposition of the detected spatial entanglement can also be done analytically, but only under the condition that the far-field (angular) radius of the SPDC ring(s) is much larger than both the angular ring width and the angular size of the detecting apertures (this is often the case in practical experiments). This assumption allows one to linearize the momentum mismatch and use a similar Gaussian decomposition as before. We will perform this exercise only for the noncollinear type-I geometry [Fig. 1(c)]; the type-II geometries [Figs. 1(b) and 1(d)] can be solved in an analogous way.

For the noncollinear type-I geometry, Fig. 1(c) depicts how we linearize the transverse momenta of the signal and idler around their average momenta via $q_{x}=q_{0}+\delta q_{x}$ and $k_{x}=-q_{0}+\delta k_{x}$. As the ring radius was assumed to be relatively large $\left(q_{0} \gg \delta q_{x}, \delta k_{x}, q_{y}, k_{y}\right)$ we can locally approximate the curved ring by a straight line and write $|\mathbf{q}-\mathbf{k}|^{2}$ $\approx 4 q_{0}\left(\delta q_{x}-\delta k_{x}\right)$. If we again neglect birefringence and use a Gaussian approximation for the mismatch function we now obtain the expression $C_{P M}(\mathbf{k}, \mathbf{q}) \approx \exp \left(-b_{q}^{2}\left|\delta k_{x}-\delta q_{x}\right|^{2}\right)$, with $b_{q}=4 b^{2} q_{0}$ as the "inverse ring width." While this linearization clearly breaks the symmetry between the $x$ and the $y$ directions, it fortunately still allows for factorization. After multiplication with the pump profile $\mathcal{E}_{p}(\mathbf{k}+\mathbf{q})$ and the aperture function $C_{a p}(\mathbf{k}, \mathbf{q})$ we find two different contributions to the Schmidt number for the decompositions in the $x$ and $y$ directions. In the weak-focusing limit, we obtain a usable or detectable two-dimensional Schmidt number of $K=K_{x} K_{y}$, where

$$
\begin{gathered}
K_{x} \approx \frac{1}{2 \sigma \sqrt{b_{q}^{2}+a^{2} / 2}}, \\
K_{y} \approx \frac{1}{a \sigma \sqrt{2}} .
\end{gathered}
$$

The associated fundamental beam waists are $\gamma_{x}^{2}$ $=2 \sqrt{2 a^{2}+4 b_{q}^{2}} / \sigma$ and $\gamma_{y}^{2}=2 \sqrt{2}(a / \sigma)$, respectively. As these are generally different, the Hermite-Gaussian modes and not the Laguerre-Gaussian modes form the preferred basis for noncollinear phase matching. The $x$ and $y$ contributions are very different when the aperture radius lies between the ring radius and ring width $\left[\left(1 / q_{0}\right) \ll a<b_{q}\right]$. This difference dis- appears when the aperture radius becomes smaller than the ring width $\left(a>b_{q}\right)$. The two-dimensional Schmidt numbers in these two cases scale as $K \propto 1 /\left(a b_{q}\right)$ and $K \propto 1 / a^{2}$, respectively. It is no coincidence that the same scaling applies to the useful apertured area of the SPDC ring.

\section{SCHMIDT NUMBER AND ETENDUE}

As an alternative, "heuristic" way to treat all four geometries in Fig. 1, we note that there is an intimate relation between the Schmidt decomposition of the two-photon SPDC field $|\Psi\rangle$ and the mode contents of the incoherent emission at the one-photon level, characterized by the density matrix $\rho_{1}$ :

$$
\begin{gathered}
|\Psi\rangle=\sum_{n=0}^{\infty} \sqrt{\lambda_{n}}\left|u_{n}\right\rangle\left|v_{n}\right\rangle, \\
\rho_{1}=\operatorname{Tr}_{v}|\Psi\rangle\left\langle\Psi\left|=\sum_{n=0}^{\infty} \lambda_{n}\right| u_{n}\right\rangle\left\langle u_{n}\right| .
\end{gathered}
$$

Instead of analyzing the modal properties of the coherent two-photon field, we can just as well decompose the incoherent one-photon field, where each coefficient $\lambda_{n}$ quantifies the (average) intensity in mode $\left|u_{n}\right\rangle$. This decomposition is well known for classical optical systems with hard-edged apertures. The "light-gathering power" of such systems is quantified by the etendue or geometric extent, which is defined as the product of the near-field beam area $A$ and its far-field opening angle $\Omega$ [15]. Normalized to the optical wavelength $\lambda$ the etendue yields the approximate number of transverse modes supported by the instrument, $N=A \Omega / \lambda^{2}$ [16]. As our Eq. (7b) is just a soft-edged version of this description, with a Gaussian pump profile in the near field and a Gaussian aperture in the far field, we expect that $N \approx K$.

Our direct comparison between the (two-photon) Schmidt number and the (one-photon) etendue might seem somewhat surprising: One could argue that (i) all generated photons are equal and contain the same information about their place of creation, and (ii) even apertured detection might not reduce this information too much as diffraction can spread the optical wave to large angles and thereby address many modes. Both arguments, however, ignore that important information is lost by spatial filtering. Photon positions at the crystal's end facet can only be known to within the diffraction limit set by the apertures. If this limit surpasses the walk-off distances in the crystal, either intrinsic due to birefringence or extrinsic due to the finite observation angle, the dimensionality of the entanglement must necessarily be reduced.

That the normalized etendue $N$ and the Schmidt number $K$ are indeed roughly equivalent is easily shown for the collinear type-I case. Substituting the pump width $w=2 / \sigma$ in the pumped area $A=\pi w^{2}$ and the angular width of the SPDC profile $\Theta=\lambda /(4 \pi b)$ in the space angle $\Omega=\pi \Theta^{2}$, gives as mode number $N=A \Omega / \lambda^{2}=1 /(2 b \sigma)^{2}$. This is indeed identical to the calculated Schmidt number $K$ in the weak-focusing limit [23]. 
Using the relation $K \approx N$, we can also easily characterize other geometries. As a first example we again consider the change from the collinear to the noncollinear geometry. The corresponding change in detected entanglement was found above to depend on the aperture size. If the detecting aperture is approximately matched to the ring diameter $\left(a \approx b_{q}\right)$, the Schmidt number of the detected entanglement in the noncollinear geometry will be approximately a factor $b^{2} / b_{q}^{2}$ smaller than that of the generated entanglement in the collinear geometry. This shows the effect of spatial filtering on the Schmidt number.

As a numerical example, we consider the noncollinear type-II geometry used in [8]. With a typical ring radius of $50 \mathrm{mrad}$, a ring width of $5 \mathrm{mrad}$, and a pump-beam divergence of $0.5 \mathrm{mrad}$, the normalized etendue associated with the pumped area and with the space angle spanned by the full SPDC rings is $\approx 10^{4}$. The number of entangled modes detected behind $5 \mathrm{mrad}$ apertures is, however, much smaller, being (only) $\approx 10^{2}$.

As a second numerical example we consider the collinear type-II geometry employed by Sullivan et al. [12]. For this geometry one should again use the linearized expressions presented in this paper and not the earlier results from Ref. [14]. As $b_{q} \gg b$, the number of modes detected in the experiment is necessarily much smaller than the value calculated from the collinear expression. This explains the discrepancy mentioned in Ref. [12], where the authors estimate a mode number of $N=16$ in position space, whereas they calculate $K \geq 360$ from the expression in Ref. [14].

In comparing the analytic expressions reported in this paper with experimental results we recall that we have used two major simplifications: the sinc-type phase-matching functions were approximated by Gaussian functions and the apertures were assumed to have a Gaussian transmission profile. For this reason, the comparison between experiment and theory can be no better than within factors like $\pi / 2$ and $\sqrt{2}$. For the same reason we have chosen to roughly approximate both the $\operatorname{sinc}(x)$ and $\operatorname{sinc}\left(x^{2}\right)$ functions by the same function $\exp \left(-x^{2}\right)$, without the refined $x$ scaling that has been used by other authors [24].

\section{CONCLUDING DISCUSSION}

In conclusion, we have presented analytic Schmidt decompositions for the spatial entanglement generated in both collinear and noncollinear SPDC. A crucial role was played by the expansion in Eq. (2), which was inspired by Snider's result in collision physics [18] and which might be useful also in a broader context. We have introduced the distinction between the generated and detected or usable numbers of entangled modes and quantified the role of the practically inevitable apertures in the detection system. The suggested link between the two-photon Schmidt number and the classical etendue allows for simple estimates of the effective number of transverse modes in various geometries.

\section{ACKNOWLEDGMENTS}

We acknowledge support of the "Stichting voor Fundamenteel Onderzoek der Materie (FOM)" and the EU program ATESIT.
[1] J. S. Bell, Physics (Long Island City, N.Y.) 1, 195 (1964); A. Aspect, P. Grangier, and G. Roger, Phys. Rev. Lett. 47, 460 (1981)

[2] H. Bechmann-Pasquinucci and A. Peres, Phys. Rev. Lett. 85, 3313 (2000).

[3] A. Ekert and P. L. Knight, Am. J. Phys. 63, 415 (1995).

[4] P. G. Kwiat, K. Mattle, H. Weinfurter, A. Zeilinger, A. V. Sergienko, and Y. Shih, Phys. Rev. Lett. 75, 4337 (1995).

[5] A. Mair, A. Vaziri, G. Weihs, and A. Zeilinger, Nature (London) 412, 313 (2001); S. S. R. Oemrawsingh, A. Aiello, E. R. Eliel, G. Nienhuis, and J. P. Woerdman, Phys. Rev. Lett. 92, 217901 (2004).

[6] J. P. Torres, Y. Deyanova, L. Torner, and G. Molina-Terriza, Phys. Rev. A 67, 052313 (2003); M. Barbieri, F. De Martini, G. Di Nepi, and P. Mataloni, Phys. Rev. Lett. 92, 177901 (2004).

[7] S. P. Walborn, S. Padua, and C. H. Monken, Phys. Rev. A 71, 053812 (2005).

[8] S. S. R. Oemrawsingh, X. Ma, D. Voigt, A. Aiello, E. R. Eliel, G. W. 't Hooft, and J. P. Woerdman, Phys. Rev. Lett. 95, 240501 (2005).

[9] A. Aiello, S. S. R. Oemrawsingh, E. R. Eliel, and J. P. Woerdman, Phys. Rev. A 72, 052114 (2005).

[10] N. K. Langford, R. B. Dalton, M. D. Harvey, J. L. O'Brien, G. J. Pryde, A. Gilchrist, S. D. Bartlett, and A. G. White, Phys. Rev. Lett. 93, 053601 (2004).
[11] R. S. Bennink, S. J. Bentley, R. W. Boyd, and J. C. Howell, Phys. Rev. Lett. 92, 033601 (2004).

[12] M. N. O'Sullivan-Hale, I. A. Khan, R. W. Boyd, and J. C. Howell, Phys. Rev. Lett. 94, 220501 (2005).

[13] L. Neves, G. Lima, J. G. Aguirre Gómez, C. H. Monken, C. Saavedra, and S. Pádua, Phys. Rev. Lett. 94, 100501 (2005).

[14] C. K. Law and J. H. Eberly, Phys. Rev. Lett. 92, 127903 (2004).

[15] F. G. Smith and T. A. King, Optics and Photonics (Wiley, New York, 2000), p. 248.

[16] A. Yariv, Quantum Electronics, 3rd ed. (Wiley, New York, 1989), p. 572.

[17] C. H. Monken, P. H. Souto Ribeiro, and S. Padua, Phys. Rev. A 57, 3123 (1998).

[18] R. F. Snider, Phys. Rev. A 33, 178 (1986).

[19] J. Keilson and K. E. Storer, J. Appl. Math. 10, 243 (1952).

[20] P. R. Berman, J. E. M. Haverkort, and J. P. Woerdman, Phys. Rev. A 34, 4647 (1986).

[21] M. H. Rubin, Phys. Rev. A 54, 5349 (1996).

[22] P. S. K. Lee, M. P. van Exter, and J. P. Woerdman, Phys. Rev. A 72, 033803 (2005).

[23] Rewritten in a different form that is more useful for experiments, $N=K=2 z_{p} / L$, where $z_{p}$ is the Rayleigh range of the pump inside a crystal of length $L$.

[24] A. Joobeur, B. E. A. Saleh, T. S. Larchuk, and M. C. Teich, Phys. Rev. A 53, 4360 (1996). 